nischen Verbindung dem Organismas einverleibt wird, sich in dem Harn nur als anorganisches Salz ausscheidet.

Aus dem vorher Angeftihrten geht hervor, dafs die genannten Methoden in der Ausführung verhältnismälsig einfach sind, sehr ubereinstimmende Resultate liefern und dieselben daber ohne Zweifel in der Praxis Beachtung finden durften.

M a r b u r g, im August 1893.

Aus dem pharmakologischen Institut der Universität Bonn.

\title{
Ueber das Additionsprodukt von Pyridin mit Monochloraceton.
}

Von Dr. med. H. D reser, Privatdozent und Assistent.

(Eingegangen den 14. März 1894.)

Von pharmakologischen Gesichtspunkten ausgehend, beabsichtigte ich die Wirkungen eines Alkaloides zu untersuchen, welches zugleich Ammoniumbase und anch Keton ist. Die beiden Körper Monochloraceton und Pyridin addieren sich zu einer Ammoniumbase ebenso wie Jodmethyl und Pyridin ;

$$
\mathrm{C}_{3} \mathrm{H}_{3} \mathrm{~N}+\mathrm{ClCH} 2-\mathrm{CO}-\mathrm{CH}_{3}=\mathrm{C}_{5} \mathrm{H}_{3} \cong \mathrm{N}<\mathrm{CH}_{2}-\mathrm{CO}-\mathrm{CH}_{3}
$$

Da, wie ich aus Beilstein's Handbuch der organischen Chemie ersehe, diese Verbindung noch nicht beschrieben ist, futhre ich hier kurz die Darstellung und einige Salze an.

Mischt man in molekularem Verhältnis Monochloraceton (Mol. Gew. 92,5) und Pyridin (79), so ist gewöhnlich nach längstens 24-stündigem Stehen bei Zimmertemperstur die Flüssigkeit zu einem Krystallkuchen erstarrt, der eine gelbliche bis gelbbraune Farbe besitzt. Kleine Reste von unverbundenem Pyridin und Monochloraceton sìnd noch eingeschlossen. Erwärmt man dagogen das Cto misch auf dem Wasserbado so orfolgt die Reaktion awar unmittelbar, aber die ointretende dunkelbranne Färbung bedingt wogen der nachfolgenden Reinigungsoperationen leicht exhebliche Verluste.

Die Krystalle, welehe das Chlorhytrat der neuen Ammoninmbase vorstellen, sind aulserst hygroakopisch. Das bei Zimemertempo- 
ratur entstandene gelbliche Produkt läßst sich leicht weils erhalten, indem man es mehrfach mit Aether, der mit Chlorcalcium entwässert ist, bearbeitet; die gelbe Verunreinigung, das noch unveränderte Pyridin und Monochloraceton werden damit entfernt und nach dem Trocknen in dem von Prof. H. Me yer angegebenen Trockenapparat (im Vacuum, über $\mathrm{H}_{2} \mathrm{SO}_{4}$ und gleichzeitig bei $100^{\circ}$ ) erhält man die weilse Krystallmasse gewichtskonstant.

Eine Chlorbestimmung gab folgende Zahlen:

$1,2818 \mathrm{~g}$ des salzsauren Salzes gaben $1,0722 \mathrm{AgCl}=0,2652 \mathrm{Cl}$

$\mathrm{Cl}$ in $\mathrm{C}_{8} \mathrm{H}_{10} \mathrm{NO} . \mathrm{Cl}$ berechnet $20,65 \%$, gefunden $20,69 \%$.

Zur Reinigung des durch Krwärmen gewonnenen braungefärbten Reaktionsproduktes empfahl sich besonders die Fallung der zuvor durch Erhitzen auf dem Wasserbade von Monochloraceton befreiten wässerigen Lösung mit Quecksilberchlorid. Krystallisiert man dieses Quecksilberdoppelsalz aus siedendem Wasser um, so sammeln sich alle Verunreinigungen auf dem Boden der Porzellanschale als braunes siegellackartiges Harz, die siedend filtrierte Flüssigkeit scheidet schwach gelbliche Krystallnadeln ans, die nach nochmaligem Umkrystallisieren rein weifs sind. -- Zur Analyse wurde dieses Quecksilberchloriddoppelsalz nochmals aus heifsem Alkohol umkrystallisiert; es besafs den Schmelzpunkt $119^{\circ} \mathrm{C}$. (unkorrig.) und eine $\mathrm{Hg}-\mathrm{Be}-$ stimmung gab folgende Zahlen:

$0,7905 \mathrm{Hg}$-Doppelsalz gaben $0,4161 \mathrm{Hg} \mathrm{S} *)=0,3587 \mathrm{Hg}$

$\mathrm{Hg}$ im $\mathrm{C}_{8} \mathrm{H}_{10} \mathrm{NOCl} . \mathrm{Hg} \mathrm{Cl}_{2}$ berechnet $45,19 \%$, gefunden $45,38 \%$.

Zur Darstellung weiterer Verbindungen wurden die Krystalle des gereinigten $\mathrm{Hg}$-Doppelsalzes fein zerrieben in Wasser suspendiert und mit $\mathrm{H}_{2} \mathrm{~S}$ zerlegt. Nach dem Verjagen des $\mathrm{H}_{2} \mathrm{~S}$ wurde das Filtrat unter Zusatz von etwas Alkohol eingeengt und dann mit Pikrinsäure, Platinchlorid und Goldchlorid gefällt. - Das aus heilsem Wasser krystallisierte Pikrat bildet schöne Prismen vomSchmelz-

*) Anmerk.: Die Ausfăllung des Schwefelquecksilbers wurde in der Weise bewirkt, dafs das sehr schwer lösliche Alkaloiddoppelsalz zunächst durch uberschlissiges Alkalihyposulfit in Lösung übergeftihrt und durch Ammoniak alkalisiert wurde, wobei nichts unlöslich ausfallt. Die erste Blase $H_{2} \mathrm{~S}$, welche in derartige Lösungen eintritt, scheidet sofort s chwarzos HgS aus, nicht die bekannten weifsen. dann gelb und braun werdenden Niederschlage. Der sonst dem HgS sich anhaftende Schwefel wird von dem Einfach-Schwefelammonium sofort aufgenonamen. Die nachträgliche Behandlung des $\mathrm{HgS}_{\mathrm{S}} \mathrm{mit}$ $\mathrm{Na}_{2} \mathrm{SO}_{3}$ oder $\mathrm{CS}_{2}$ ist dadurch ganz uberflussig geworden 
punkte $141^{\circ}$ C. unkorrig. Das zum Vergleich dargestellte Pyridinpikrat schmolz bei $163^{\circ} \mathrm{C}$.

Von dem Pikrat machte ich auch C.- und H.-Bestimmungen durch Verbrennen mit $\mathrm{CuO}$ und vorgelegter blarker Cu-Drahtnetzrolle :

I. 0,5990 Substanz gaben $1,0149 \mathrm{CO}_{8}=0,2768 \mathrm{C}$

$$
0,1767 \mathrm{H}_{2} \mathrm{O}=0,0196 \mathrm{H}
$$

Die Formel $\mathrm{C}_{8} \mathrm{H}_{10}$ NO . $\mathrm{C}_{6} \mathrm{H}_{2} \mathrm{~N}_{3} \mathrm{O}_{7}$ verlangt $46,15 \%$ C, gef. $46,21 \%$

II. 0,5459 Substanz gaben $0,9222 \mathrm{CO}_{2}{ }^{n}=0,2515 \mathrm{C}$ $3,29 \%$ H, $\quad 3,27 \%$ $0,1602 \mathrm{H}_{8} \mathrm{O}=0,0178 \mathrm{H}$ verlangt $46,15 \%$ C, gef. $46,07 \%$ $\Rightarrow 3,29 \% \mathrm{H}, " 3,26 \%$

Pyridinpikrat $\mathrm{C}_{5} \mathrm{H}_{5} \mathrm{~N} . \mathrm{C}_{6} \mathrm{H}_{3} \mathrm{~N}_{3} \mathrm{O}_{7}$ verlangt $42,85 \% \mathrm{C}$ und $2,59 \% \mathrm{H}$. 0,4960 Substanz gaben $0,7898 \mathrm{CO}_{2}=0.2154 \mathrm{C}$ gef. $43,42 \% \mathrm{C}$. $0,1142 \mathrm{H}_{2} \mathrm{O}=0,0127 \mathrm{H}, \quad 2,56 \% \mathrm{H}$. Es ergiebt sich aus diesen Zahlen, dafs die Ketongruppe mit dem Pyridinmolekül in feste Verbindung getreten ist. Ihre Anwesenheit lärst sich ferner leicht durch die Farbenreaktion mit Nitroprussidnatrium darthun; man setzt zu der wässerigen Lösung des salzsauren Salzes etwas Nitroprussidlösung (verdünnt, trisch bereitet); auf Zusatz von Kali- oder Natronlauge wird die Lösung dunkelrot; Nitroprussidnatrium allein wird mit Lauge gelb. Aufserdem reagierte die Lösung des salzsauren Salzes im Wasserbad, mit essigsaurem Natrium und salzsaurem Phenylhydrazin erhitzt, bereits nach 1/4 Stunde unter Abscheidung eines Hydrazons in Form gelber, öliger Tropfen.

Das Platinchloriddoppelsalz ist in kaltem Wasser schwer löslich, aus heifsem krystallisiert es in kleinen Prismen; es schmilzt bei $206-2070$ C. (unkorrig.) unter Gasentwickelung mit Zersetzung während Pyridinchloroplatinat erst bei 240-242 ${ }^{\circ}$ schmilzt.

Eine Gluhbestimmung ergal, aus 0,4431 Pt.-Doppelsalz 0,1270 Pt. gleich 28,66 Proz. Die Formel $\left(\mathrm{C}_{8} \mathrm{H}_{10} \mathrm{NO} \mathrm{Cl}\right)_{2}$. $\mathrm{Pt} \mathrm{Cl}_{4}$ verlangt 28,60 Proz.

Das Goldchloriddoppelsalz ist ebenfalls in kaltem Wasser sehr schwer löslich, aus heilsem, mit Salzsäure versetztem Wasser kann es in dünnen, oft $1 \mathrm{~cm}$ langen Nadeln erhalten werden; Schmelzpunkt $136-138^{\circ}$ C. (unkorrig.)

Eine Glühbestimmung ergab aus 0,4503 Au-Doppelsalz 0,1852 Au gleich 41,13 Proz. Au. Die Formel $\mathrm{C}_{8} \mathrm{H}_{10} \mathrm{NOCl}$. Au $\mathrm{Cl}_{3}$ verlangt 41,3i Proz. Au. 
Nach diesen günstigen, mit dem Pyridin erhaltenen Ergebniseen versuchte ich die gleiche Reaktion mit Chinolin. Reaktion erfolgte erst durch Erwärmen auf dem Wasserbade, es bildete sich aber nur ein dunkelbrauner, dicker Syrup; nach dem Auskochen mit Wasser wurde die braune Lösung mit Quecksilberchlorid ausgefällt, das durch Umkrystallisieren gereinigte $\mathrm{Hg} \mathrm{Cl}_{2}$-Doppolsalz mit $\mathrm{H}_{2} \mathrm{~S}$ zerlegt, eingeengt, mit $\mathrm{Pt} \mathrm{Cl}_{4}$ gefällt; das umkrystallisierte Pt-Salz enthalt 28,98 Proz. Pt; das synthetische Produkt hătte 25,18 Proz. verlangt, während Chinolinplatinchlorid 29,48 Proz. verlangt. Als Schmelzpunkt des letzteren wird angegeben $218^{\circ} \mathrm{C}$.; das aus dem Reaktionsgemisch isolierte schmolz bei $212^{\circ} \mathrm{C}$.; es scheint also sich nur um unverändertes, nicht mehr ganz reines Chinolin gehandelt zu haben, wăhrend die Hauptmagse in ein nicht näher falsbares Produkt übergegangen war.

Es scheint also das Monochloraceton nur mit dem Pyridin glatt reagieren zu könneu.

\section{Mitteilungen aus dem pharmaceutisch-chemischen Institute der Universität Marburg}

Von Ernst Schmidt.

\section{Ueber Isobernsteinsäure und Isoäpfelsäure}

von Dr. Max P u ch aus Dessau.

(Eingegangen den 13. Februar 1894.)

\section{Isobernsteinsäure.}

Auf die Existenz einer mit der Bernsteinsäure oder Äthylendikarbonsäure isomeren Säure, einer $\ddot{A} \mathrm{th}$ yli d endikarbonsäure:

$$
\begin{aligned}
& \mathrm{CH}_{2}-\mathrm{CO} . \mathrm{OH} \\
& \mathrm{CH}_{2}-\mathrm{CO} . \mathrm{OH}
\end{aligned}
$$

Aethylendicarbonellnre<smiles>CCC(C(=O)O)C(=O)OO</smiles>

Aetbylidendicarbons ure

machte zuerst $\mathrm{W}$ ich $\theta \mathrm{h} \mathrm{h}_{\mathrm{h}} \mathrm{B}^{1}$ ) aufmerksam, indem or nachwies, dafs eine von H. M ïlle ${ }^{2}$ ) aus dem Ulrich'schen [a-l Chlorpropionsăureăther dargestellte und als gewöhnliche Bernsteinsăure angesprochene Säure mit letzterer nicht identisch sein könne, da sie

1) Z. f. Chem. 1867, 247.

2) Annal. d. Chem. u. Pharm. 131, 350. 